\title{
Los derechos humanos de las personas en situación de discapacidad en Colombia: estado actual y retos para el ejercicio profesional
}

\author{
Human rights of people in disability situation in Colombia: Current status and \\ challenges for professional exercise
}

*Tania Collazos Rodríguez

\section{Resumen}

Este artículo presenta una revisión de distintos documentos, tanto internacionales como nacionales, en torno al tema de los Derechos Humanos de las personas con discapacidad. Entre ellos, la Convención sobre los Derechos Humanos de las personas con discapacidad, como el tratado más importante celebrado en el siglo XXI, respecto a la defensa y protección de los DD.HH. de cerca de un $10 \%$ de la población mundial; el Protocolo Facultativo como instrumento jurídico internacional adicional a la Convención, que permite que los individuos y grupos de los Estados que lo hayan ratificado, puedan presentar sus denuncias y peticiones; y el informe que emitió el Comité de la ONU frente a la implementación del tratado en Colombia en el 2016. El análisis de esta documentación plantea la urgencia de un cambio de paradigma, acerca de la discapacidad en el país y propone retos significativos desde la disciplina psicológica para el ejercicio profesional.

Palabras Clave: Derechos Humanos, discapacidad, exclusión social.

\section{Abstract}

This article presents a review of different international and national documents on the subject of the Human Rights of persons with disabilities. These include, the Convention on the Human Rights of persons with disabilities, as the most important treaty of the twenty-first century on the defense and protection of human rights of about $10 \%$ of the world's population; The Optional Protocol as an additional international legal instrument to the Convention, which allows individuals and groups of States that have ratified it to present their denunciations and petitions; and the report issued by the UN Committee on the implementation of the treaty in Colombia in 2016. The analysis of this documentation raises the urgency of a paradigm shift about disability in the country and proposes significant challenges from the psychological discipline for professional practice.

Keywords: Human Rights, disability, social exclusion.

* Psicóloga, Universidad del Valle. Magíster en Psicología, Universidad de San Buenaventura Cali. Investigador Grupo Lumen Humanitas. Fundación Universitaria Católica Lumen Gentium. tcollazos@unicatolica.edu.co 


\section{La Convención sobre los derechos de las personas} con discapacidad

La necesidad mundial de promoción y protección de los Derechos Humanos de las personas en situación de discapacidad, fue el motor para la formulación del primer tratado internacional celebrado en el siglo XX, conocido como La Convención sobre los derechos de las personas con discapacidad, cuyo propósito es garantizar los derechos civiles, políticos, sociales y económicos de más de 650 millones de personas en el mundo, equivalente a un $10 \%$ de la población mundial (González, 2008).

Este documento comprende el término discapacidad, como aquella situación permanente o transitoria bajo la cual ciertas personas presentan alguna deficiencia física, mental, intelectual o sensorial que, a largo plazo, afecta la forma de interactuar y participar plenamente en la sociedad. Es necesario aclarar que es un concepto que evoluciona y que, actualmente, es interpretado bajo un nuevo paradigma, el cual sugiere que es una condición resultante de la interacción entre las personas con determinada deficiencia y las barreras que encuentra debido a la actitud y al entorno, y que evitan la participación plena y efectiva de ellas en la sociedad, en igualdad de condiciones que los demás (González, 2008).

La Convención sobre los derechos de las personas con discapacidad, llamada en adelante La Convención, se fundamenta sobre la base de los principios de la Carta de las Naciones Unidas, la Declaración Universal de los Derechos Humanos y en los Pactos Internacionales de Derechos Humanos, cuyo propósito fundamental es el reconocimiento de la dignidad y los valores inherentes de los derechos iguales e inalienables de todo ser humano.

Este tratado determina una serie de lineamientos y reglamentaciones en torno a la salud, la educación, la accesibilidad, la libertad de movimiento, el empleo, la habilitación y rehabilita- ción, la participación en la vida política, la igualdad y la no discriminación.

En este sentido, la Convención constituyó un momento importante para 192 países que participaron en la reflexión, discusión y toma de decisiones, en beneficio de una población con necesidades de apoyo importantes en todo el mundo. Especialmente, para la mayoría que vive en países que no tienen fácil acceso a servicios médicos. Esto pone en relieve la relación entre la pobreza y la discapacidad, en tanto que las personas tienen un mayor riesgo de deterioro de su situación de discapacidad cuando encuentran en un estado de pobreza, ya que demandan más recursos para su efectiva atención (Hernández, 2007).

Durante la formulación del tratado se tomaron posiciones de vanguardia en temas claves como la salud sexual y reproductiva, el hogar y la familia, y la necesidad de monitoreo internacional para verificar la implementación de los derechos de la Convención en los países participantes (González, 2008). Así mismo, se hizo un llamado al Estado y a la sociedad sobre el papel que ocupa la familia como unidad colectiva natural que tiene derecho a recibir su protección y asistencia para garantizar una real igualdad de condiciones y el goce pleno de los derechos de las personas en situación de discapacidad.

Por tanto, la Convención hace un llamado a los Estados parte a garantizar su implementación en distintos ámbitos de participación, tales como la cultura, la educación, el ámbito jurídico, administrativo y presupuestal. En el documento se abordan temas de sumo interés para la protección de los derechos humanos de las personas en situación de discapacidad, por lo que vale la pena mencionar brevemente aquellos artículos que serán útiles para su posterior análisis, en materia de protección e implementación en el Estado colombiano.

La Convención tiene como principal objetivo "promover, proteger y asegurar el goce pleno y en condiciones de igualdad de todos los derechos humanos y libertades fundamentales por todas las per- 
sonas con discapacidad, y promover el respeto de su dignidad inherente" (Convención sobre los derechos de las personas con discapacidad, 2007, p. 4).

En su artículo $3^{\circ}$ se propone, fundamentalmente, el respeto por la autonomía individual, incluyendo la libertad de tomar las propias decisiones, la independencia de las personas, el derecho a participar de forma plena y efectiva en la sociedad, el respeto por la diferencia y la aceptación de esta población como parte de la diversidad y de la condición humana, el respeto al desarrollo de las facultades de los niños en situación de discapacidad, y de su derecho a preservar su identidad.

Por otra parte, menciona en su artículo 8o, la importancia de la toma de conciencia para que las sociedades tengan una actitud de inclusión y valoración hacia las personas en situación de discapacidad. El artículo $9^{\circ}$ retoma el tema de acceso a nuevas tecnologías, lo que implica que las personas encuentren formas efectivas de comunicación y acceso a la información digital.

Es necesario dedicar especial atención al artículo $12^{\circ}$ de la Convención, referido al reconocimiento de las personas como sujetos con capacidad jurídica, aunque las personas con discapacidad requieran apoyo para ejercerla. Con relación a esto, González (2008) comenta que "El verdadero punto que se discutió, fue la prescripción de las salvaguardas que serían adoptadas para evitar el abuso en el ejercicio de la capacidad legal de las personas con discapacidad y su temporalidad" (p. 534). Con relación a este artículo, el Informe Mundial sobre el derecho a decidir Independiente pero no Solo (Laurin-Bowie, 2014), expone el punto de vista de las personas en situación de discapacidad de carácter intelectual, pertenecientes a distintos países del mundo, sus cuidadores y familias frente a su derecho a decidir. Este informe, identifica un principio fundamental denominado autodeterminación, definido como "el concepto de que todas las personas deben tener el control de las decisiones que afectan su vida" (Lázaro et al., 2014, p. 14), lo cual propone que para que una persona en situación de discapacidad intelectual ejerza el derecho a decidir y tome decisiones de carácter legislativo, es necesario promover reformas legislativas, transformaciones en los mecanismos de apoyo, en las políticas públicas y el desarrollo, en los procesos en favor de la toma de decisiones con apoyo y, sobretodo, un cambio de actitud frente a la mirada de una persona en condición de discapacidad.

Retornando a la Convención, del artículo $25^{\circ}$ al $30^{\circ}$, se destacan temas puntuales como el derecho al empleo, a la recreación, la participación cultural y la salud. Y para terminar, se cita el artículo $32^{\circ}$, el cual produjo gran controversia, ya que hace referencia a la cooperación internacional y la destinación de recursos de apoyo de los países económicamente más favorecidos. Lo que parece preocupar a estos Estados es que, en vista de que ya poseen leyes que salvaguardan la población en cuestión, consideran que un tratado de esta índole vendría a ser un mecanismo de defensa más al ciudadano, para que demande al Estado con "nuevos" derechos (González, 2008).

Hasta aquí, una breve presentación de asuntos de relevancia de la Convención que reflejan la dificultad que tienen las personas con discapacidad para asumir sus vidas, además de las que ya tienen las personas que cuentan con condiciones regulares, puesto que enfrentan desafíos que deben resolver con herramientas que superan su propia voluntad y que exigen la participación de todo un entramado social. Por lo tanto, es un tratado que exhorta a la acción institucional de los Estados parte al ejercicio de la justicia y la democracia, como un requerimiento ético en tiempos de proclamación de la igualdad (Sanjosé, 2006).

\section{El Protocolo Facultativo de la Convención sobre los derechos de las personas con discapacidad}

El Protocolo Facultativo es un instrumento jurídico internacional independiente, adicional a la Convención. Ambos se concretaron en el 2008, debido al urgente llamado de las personas con dis- 
capacidad de todo el mundo, con el fin de que se protegieran y respetaran sus derechos humanos, en igualdad de condiciones (Organización de las Naciones Unidas [ONU], 2014).

El Protocolo Facultativo permite que los individuos y grupos de los países que lo hayan ratificado, puedan presentar sus denuncias y peticiones ante el Comité de los derechos de las personas con discapacidad, liderado por las Naciones Unidas, una vez que se hayan agotado todos los procesos y recursos posibles en su país (ONU, 2008). En otras palabras, los Estados parte del Protocolo Facultativo reconocen al Comité como el organismo competente para revisar dichas denuncias, reafirmar que sus derechos han sido vulnerados y hacer recomendaciones a los Estados.

La Convención actualmente se encuentra ratificada por 173 Estados parte y con 92 que ratificaron el Protocolo Facultativo, donde Colombia es uno de los Estados que, a la fecha, no ha firmado ni ratificado el Protocolo Facultativo (ONU, 2017).

Vale la pena reconocer la labor de la Organización de las Naciones Unidas en su propósito de proteger y hacer valer los derechos humanos que enmarcan las condiciones para una vida digna de las personas en situación de discapacidad, en la sociedad de la cual hacen parte. Por tanto, es necesario que como una acción que denote verdadera valoración a esta significativa población, el Estado colombiano socialice, de manera efectiva, a sus ciudadanos la participación en el tratado de la Convención, su contenido, estimule la reflexión de los derechos que allí se exponen, y los diversos mecanismos legislativos de apoyo con los que el ciudadano cuenta para su implementación.

De igual modo, es recomendable generar espacios colectivos para la discusión de la participación del Estado colombiano en los tratados, donde, por ejemplo, se expongan públicamente su no participación en el Protocolo Facultativo y de las implicaciones para la población en situación de discapa- cidad en no contar con mecanismos de denuncias individuales de la Convención.

\section{Los niños con discapacidad en Colombia: una po- blación doblemente vulnerable}

La vulnerabilidad está relacionada con causas externas (evolución del mercado de trabajo, recortes en los recursos de protección social, inseguridad ciudadana, deterioro ambiental, clima social, etc.), pero también con la apreciación e interiorización subjetiva de la propia vulnerabilidad (incertidumbre, miedo, sensación de inseguridad, pérdida de autoestima y confianza en las propias capacidades, etc.) (Lázaro et al., 2014, p.18). Por tanto, se reconoce como una experiencia que abarca la dimensión psicológica y social de una persona.

Respecto a Colombia, el reporte de la niñez colombiana en cifras, presentado por el Fondo de las Naciones Unidas para la Infancia, UNICEF (2002), informa con todo detalle la situación de la población más vulnerable del país: los niños. Esta información es ratificada por la Convención en su artículo 6 y 7, la cual indica que las poblaciones que podrían ser más vulneradas y discriminadas en el mundo en cuanto a sus derechos, son los niños y las mujeres con discapacidad.

Todo lo anterior, pese a la existencia de un marco legal estipulado en el Código de Infancia y Adolescencia en Colombia (2006), el cual reúne las bases del jurídicas para la defensa de los derechos de los niños y adolescentes en el territorio nacional.

A continuación, se presentan apartados importantes dedicados a los derechos de los niños y adolescentes en situación de discapacidad presentes en el Código que, una vez más, como en el caso de la Convención, dejan en entredicho la efectividad del mismo, más aún cuando no son claros los mecanismos para su efectiva defensa.

En el artículo 2o del Código, expedido por la ley 1098 del 2006, se expone que el objeto del Código 
es "establecer normar sustantivas y procesarlas para la protección de los derechos de los niños y adolescentes, y garantizar el ejercicio de sus derechos y libertades consagrados en los instrumentos internacionales de Derechos Humanos, en la Constitución política y en las leyes, así como su restablecimiento" (Martin, Quijano, Gallardo, Mojica, 2007, p. 10).

El artículo 36, afirma que esta población tiene derecho a gozar de una calidad de vida plena, donde el Estado proporcione las condiciones necesarias para que puedan valerse por sí mismos. Este artículo aboga por la autonomía y el respaldo del Estado para lograr su independencia, el desarrollo máximo de sus potencialidades y el derecho a recibir atención, cuidados especiales en salud y apoyo a los miembros de la familia.

Martin et al., (2007), hacen mención a las libertades fundamentales que expone el artículo $37^{\circ}$ del Código, el cual cita que los niños y adolescentes en Colombia, gozan de las libertades consagradas en la Constitución Política y en los tratados internacionales de los Derechos Humanos, entre los que se encuentran "el libre desarrollo de la personalidad y la autonomía personal; la libertad de conciencia y de creencias; la libertad de cultos; la libertad de pensamiento; la libertad de locomoción y la libertad para escoger profesión u oficio" (p. 34).

La legislación colombiana dedica especial atención a los niños en una situación de discapacidad, población que por su propia condición de infancia, los hace doblemente vulnerables.

Sin embargo, ampliando a la población en situación de discapacidad, otras condiciones asociadas a la vulnerabilidad, como por ejemplo, el género, etnia, condición socioeconómica, etc., permiten apreciar un panorama complejo de la discapacidad en Colombia, que genera cuestionamientos importantes acerca de la real garantía de los beneficios que promulga, ya que la pesquisa documental pone de manifiesto que los derechos, desde un marco ideal de protección, no son garantía sufi- ciente para su aplicación efectiva. Se hace urgente entonces, prestar especial atención a los mecanismos reguladores de su implementación.

\section{Informe sobre la implementación de los Dere- chos Humanos de las personas con discapacidad en Colombia}

El 31 de agosto del 2016, la ONU entregó a Colombia las observaciones finales del primer informe de implementación de la convención de los derechos humanos sobre las personas con discapacidad. El comité evaluador estudió el informe presentado por Colombia y tomó en cuenta otros informes alternativos presentados por diversas organizaciones civiles, los cuales identifican como temas de mayor importancia, la capacidad jurídica, reconocimiento de los derechos sexuales y reproductivos, accesibilidad y necesidad de cambio de paradigmas frente a la discapacidad. (ONU, 2016).

Entre las observaciones más importantes del Comité de la ONU a Colombia se citan textualmente los aspectos positivos:

- La ley estatutaria 1618 de 2013 sobre personas con discapacidad.

- La Política Nacional de Discapacidad e Inclusión social, CONPES Social 166.

- $\quad$ La ley 1752 de 2015 que define el tipo penal de discriminación por motivos de discapacidad.

- La ley 1448 de 2011 para la atención, asistencia y reparación integral a víctimas de la violencia.

- Las normas que reconocen oficialmente la lengua de señas colombiana como idioma propio de las personas con discapacidad auditiva y auditiva-visual." (ONU, 2016 p. 1).

El Comité dedica 13 de las 14 páginas del informe a las principales áreas de preocupación y sus respectivas recomendaciones. A continuación se presenta una síntesis de la mayoría de puntos abordados. 
Respecto a los principios generales y obligaciones, al Comité le preocupa: que Colombia aún no ha ratificado el Protocolo Facultativo de la Convención, que asuntos relacionados con la legislación en torno al tema de la discapacidad, las limitaciones en cuanto la capacidad jurídica de las personas con discapacidad y asuntos relacionados con la esterilización, no estén en sintonía con la Convención. Así mismo, el uso de términos peyorativos para referirse a las personas con discapacidad, especialmente intelectual y psicosocial, en la legislación, jurisprudencia y otros documentos oficiales.

Preocupa la falta de acceso a procesos de consulta de políticas de las organizaciones que atienden a la población con discapacidad y que sus aportes a las mismas no se reflejen en las decisiones finales. De igual modo, que el Sistema Nacional de la Discapacidad no promueva la participación de estas organizaciones, y que los procesos de acreditación sean dispendiosos y costosos para la población rural.

Es de cuidado que aún se empleen criterios basados en el modelo médico para la localización y caracterización de esta población en el país, y que solo un $2.59 \%$ haya efectuado su registro.

En cuanto a los derechos específicos, con relación al tema de la igualdad, es de preocupación la discriminación contra niñas y mujeres con discapacidad, la falta del reconocimiento de la denegación de ajustes razonables en leyes antidiscriminación, y que el registro de las quejas presentadas carezca de detalles importantes como el género, la edad, tipo de discapacidad y etnia.

Respecto a los niños con discapacidad, se encuentra escasa información, especialmente de aquellos que están en situación de pobreza, zonas rurales y remotas. También preocupa que no haya prohibición del castigo físico a la población infantil con discapacidad.

En cuanto a las campañas públicas y privadas para la toma de conciencia, en beneficio de la población con discapacidad, como el Teletón y el Día Blanco, se consideran que son reflejo del modelo caritativo de la discapacidad.

Con relación al tema de la accesibilidad, preocupa la falta de planes nacionales para la implementación de normas para la accesibilidad, especialmente en zonas rurales, transporte público, adecuaciones físicas para servicios públicos e información y comunicación para personas con discapacidad auditiva, visual, e intelectual.

En lo concerniente al derecho a la vida, el Comité manifiesta su preocupación por informes que indican que diez personas con discapacidad fueron asesinadas y reportadas como falsos positivos. Así mismo, inquieta la escasa participación en programas para la reducción de riesgos de desastres y la poca facilidad de acceso a la información, tanto como la casi nula inclusión de esta población en los procesos de negociación de paz.

Preocupa la carencia de datos estadísticos del número de personas que están detenidas a razón de su discapacidad, haciendo énfasis en la privación de la libertad de personas con discapacidad psicosocial. Por otra parte, es inquietante que las personas privadas de la libertad no cuenten con servicios específicos de atención en salud, prestaciones en igual condición que los demás, ni hagan parte de programas para desarrollo vocacional.

Referente a la protección contra la tortura y otros tratos o penas crueles, inhumanos o degradantes, la ONU encuentra muy inquietante que en Colombia no existan medios para la prevención y protección contra la tortura, ni un aparato legal que clasifique las formas específicas de tortura que puede padecer una persona con discapacidad. Recomienda al Estado hacer parte del Protocolo Opcional de la Convención Contra la Tortura y otros Tratos Crueles, Inhumanos o Degradantes (OPCAT). 
Uno de los temas que más inquietud debe generar, son los bajos niveles de participación de la población con discapacidad en todos los niveles educativos, la discriminación y el rechazo que viven en las escuelas regulares, la falta de formación pedagógica y didáctica para su atención, y el predominio de "aulas especializadas", dentro de las escuelas regulares. Al parecer, el tema de la inclusión educativa sigue girando en torno a cuestiones de cobertura, no de procesos que aseguren la real integración y promoción de aprendizajes significativos de esta población.

Al tema de la educación, se suman las restricciones para la participación política, especialmente para aquellas personas declaradas interdictas por su condición de discapacidad.

Como preocupación final de la ONU (2016) respecto a la implementación de la Convención, manifiesta que Colombia no ha cumplido con la ley 1618, la cual hace referencia a la designación de un mecanismo independiente de monitoreo de este tratado. "Asimismo, le preocupa que la Defensoría del Pueblo tenga la defensa de los derechos de las personas con discapacidad bajo la Defensoría delegada para la Salud, la Seguridad Social y la Discapacidad, reforzando el modelo médico de la discapacidad." (p. 13)

Hasta aquí una síntesis de la mayoría de motivos de preocupación. Se recomienda revisar en detalle los motivos de intranquilidad que expone el Comité en el documento original. De igual manera, se exhorta a examinar cada una de las recomendaciones que le hacen al Estado colombiano, las cuales no se presentan en este documento, adoptando una posición crítica y reflexiva, la nuevas formas de intervención, apoyo y acompañamiento a la población en cuestión, especialmente para aquellas personas donde convergen distintas condiciones de vida que multiplican su estado de vulnerabilidad, como es el caso de las mujeres, niñas, desplazados, marginados, en situación de discapacidad.
La formulación de un tratado que apunta a la protección e implementación de los derechos humanos de las personas con discapacidad, es un punto de partida importante para visualizar tanto las necesidades y problemáticas que vive tal población y funciona como un interesante mecanismo de evaluación, donde los estados que se acogen a la Convención muestran sus resultados, planes de acción y/o proyectos ejecutados o en desarrollo, partiendo de ese marco de referencia.

Sin embargo, se evidencia que la participación como Estado parte de la Convención, es una condición necesaria, mas no suficiente para su real implementación. Es decir, que la existencia de un tratado universal que refleja de forma implícita una invitación a transformar tanto la forma de ver la discapacidad, así como la forma de definirla e intervenirla, no es garante para su aplicación en aquellos estados que la acogen.

La falta de un mecanismo de denuncias individuales de la Convención, como es el Protocolo Facultativo para las personas en situación de discapacidad, refuerza el hecho de que esta población continúe en un terreno de marginalidad y desprotección, enfrentando dificultades de base, como la mentalidad cultural asociada a los paradigmas tradicional y médico de la discapacidad.

\section{Necesidad de un cambio de paradigma}

Partiendo de la idea de que un paradigma es un sistema básico de creencias que determina una visión del mundo, con implicaciones ontológicas, epistemológicas y metodológicas que determinan una forma particular de estudio de la realidad (Guba y Lincoln, 1994), a continuación se exponen tres paradigmas que definen la discapacidad.

Desde una perspectiva del paradigma tradicional, la discapacidad se define desde el modelo de prescindencia (Palacios, 2008), dentro del cual se establece una relación con la persona con discapacidad con prácticas que segregan, marginan o exterminan y con quienes 
se establece una relación desigual, donde prevalecen actitudes como la lástima y la caridad hacia quienes se denominan "anormales", "discapacitados", "lisiados" o "minusválidos", etc.

Desde el ámbito científico, el tema de la discapacidad fue históricamente abordado por disciplinas clásicas tales como la Medicina, la Pedagogía y la Psicología, que situaron la «discapacidad» como un elemento constitutivo de la "condición de ser» de quienes tienen las discapacidades (Villa y Arnau, 2009). Este paradigma médico-biológico, interpreta las deficiencias como patologías internas del organismo que requiere de la rehabilitación para procurar un estado de normalidad al sujeto (Villa y Arnau, 2009). Las deficiencias actúan como obstáculos de la persona con discapacidad para su inclusión social y la persona es quien tiene el papel de transformarse. Por tal razón, no se contempla como necesaria la modificación, adecuación o creación de nuevos entornos para su inclusión en la sociedad a la cual pertenezca (Padilla 2010).

Si bien estos dos paradigmas siguen vigentes en diversos contextos (sociales, académicos, políticos, etc.), en las dos últimas décadas se han realizado esfuerzos para resignificar la discapacidad y orientar su conceptualización hacia un paradigma de enfoque social (Alba Pastor y Zubillaga del Río, 2012; Palacios, 2008 y Padilla, 2010; citados por Alfaro, 2013).

El paradigma social plantea que el entorno y la sociedad es quien debe propender, por una parte, por el desarrollo de las capacidades de una persona, independiente de su situación de discapacidad y, por otra, por la generación entornos adecuados, modificados o generados para la participación de las personas con una condición de funcionalidad distinta en la sociedad, pues en el momento en que las personas con discapacidad no encuentran barreras en su entorno para desarrollarse y desenvolverse como sujetos, la discapacidad deja de existir.

Aunque se hace evidente la dificultad en nuestra sociedad para aceptar al Otro como es, invirtiendo todos los esfuerzos en intentar cambiar a quien tiene condiciones distintas, al modelo dominante de supuesta homogeneidad, es decir "sin discapacidad" (Skliar, 2003), es necesario y urgente un cambio de paradigma, donde la capacidad no sea excluyente de la situación de discapacidad, pues toda persona cuenta con talentos que nacen de la diferencia y no de la deficiencia (Schorn, 2003).

Con relación a lo anterior, la adopción de un paradigma distinto al tradicional y al médico - biológico, implica nuevas formas de definir, conceptualizar e intervenir las personas en situación de discapacidad.

Romañach y Lobato (2005), plantean una propuesta que promueve un cambio de mirada que trasciende la terminología médica dominante, la cual presenta a la persona como un ser biológicamente imperfecto a quien se debe rehabilitar para que sea "normal". Los autores afirman que en tanto se utilicen términos limitantes o despectivos para denominar a ese colectivo, se ratificará su minusvaloración y, por lo tanto, se mantendrá dicha discriminación. Por tal razón, se plantea el término "diversidad funcional", el cual se ajusta a una realidad en que una persona funciona de forma distinta a la de la mayoría de la sociedad (Romañach y Lobato, 2005).

Es interesante encontrar que el paradigma social de la discapacidad se encuentra reflejado en los principios que fundamentan la Convención y su Protocolo facultativo, permanentemente aboga por la dignidad humana de las personas con discapacidad, y "cuestionan percepciones anteriores sobre la discapacidad — como problema médico o como origen de planteamientos basados en la compasión o la beneficencia- y establece, respecto de la discapacidad, un enfoque de empoderamiento que se basa en los derechos humanos" (ONU, 2014, párr. 3).

Sin embargo, el informe que emite el Comité de la ONU respecto a la implementación de los derechos de las personas con discapacidad, deja claro que el Estado colombiano es foco de mayores preocupaciones que de los aspectos positivos, lo que refleja un notable 
predominio de los paradigmas tradicional y médico de la discapacidad en nuestro país.

\section{Retos para el quehacer psicológico: a modo de condusión}

La Convención permitió que el Derecho Internacional se dotara de instrumentos orientados a prevenir el abuso y explotación de los derechos humanos de las personas con discapacidad, que no son nuevos, pero que prohíben explícitamente la discriminación en todas las esferas de participación de los individuos (Sanjosé, 2006). Sin embargo, la evaluación de su real implementación, tal como la que realizó el Comité de la ONU, no es garante suficiente de la aplicación de la norma en un contexto que presenta múltiples y serias problemáticas de exclusión y desprotección, ya que, si bien otorga un lugar relevante de reconocimiento de los no incluidos, ofreciendo un nuevo aire esperanzador y de respaldo, es prioridad iniciar un proceso de transformación de la mentalidad de la sociedad, la cual tenga sincronía con prácticas cotidianas que la soporten.

El conocimiento y comprensión de un marco de referencia global que propende por un cambio de paradigma frente a la discapacidad, así como de otros documentos nacionales que reflejan las necesidades y problemáticas más sentidas de esta población en nuestro país, es un asunto que el ejercicio profesional no se debe subestimar, ya que las orientaciones metodológicas que el psicólogo adopte en las distintas intervenciones, por ejemplo, en los proyectos de enfoque social, educativo, jurídico, etc., reflejarán la forma en que conceptualiza la discapacidad y cómo concibe el ser de quien vive esa condición, aspectos fundamentales que quedarán reflejados en su quehacer cotidiano de profesión, en su producción intelectual y, en general, en todo aquello que publique para guía y orientación. Por tanto, el psicólogo y demás profesionales de disciplinas afines, funcionan como generadores de nuevas formas de pensamiento frente a la discapacidad o, por el contrario, pueden ser justamente quienes perpetúen aquellos modelos o paradigmas que van en contravía de estas nuevas perspectivas.
En otras palabras, la elección de una forma particular del trabajo con personas en situación de discapacidad no es un proceso que ocurra al azar o que se adopte de forma ingenua. Por lo contrario, plantea serias cuestiones ontológicas, epistemológicas y metodológicas para el psicólogo que merecen especial atención, puesto que tenemos un serio compromiso ético con esa red de interrelaciones que componen la sociedad y que está en permanente movimiento y transformación.

Las personas con discapacidad son sujetos que requieren, valga la redundancia, de una sociedad que les subjetivice, conozca no solo sus necesidades y problemáticas, sino que les haga partícipes y principales protagonistas en la significación y expresión de sus propias experiencias.

Es importante que tanto los profesionales de la psicología, así como de todos aquellos cuyo campo disciplinar esté relacionado, protejan y promuevan la dignidad humana de esta población. La Federación Europea de Asociaciones de Psicólogos (EFPA, 2016), sostiene que los psicólogos y las diversas asociaciones del gremio tienen un papel fundamental en la implementación y protección de los derechos humanos, independientemente de la condición de vulnerabilidad que tenga un grupo social en determinado contexto.

La EFPA (2016), plantea que los psicólogos y sus asociaciones como actores no estatales, deben defender los derechos humanos y denunciar cuando hay amenaza o son violados, especialmente en aquellos grupos considerados vulnerables (niños, mujeres, con discapacidad, víctimas de contextos de violencia, grupos minoritarios, personas LGTBI, etc.), fomentar la protección de la privacidad con la población que atienden, participar activamente en la formulación de políticas para integrar a las personas, en vez de marginarlas o dividirlas y adoptar una mirada de su quehacer que vaya en contra de la discriminación, tanto en la palabra como en los hechos.

Lo anterior, es una invitación a que los profesionales que atienden, intervienen, estudian o investigan la población con discapacidad, sean personas que 
orienten de forma transversal a un paulatino cambio de postura de la sociedad frente a dicha población, estimulen la reflexión y el análisis de las distintas posturas y paradigmas que promueven o no la defensa de los derechos humanos en los grupos sociales con quienes se relacione, les guíe y socialice los distintos documentos nacionales e internacionales de vanguardia y promuevan, en ellos mismos y en los demás, una postura sentida capaz de reflejarse en los actos cotidianos y que dé cuenta de un mayor respeto y valoración por la diversidad humana.

\section{Referencias bibliográficas}

Alfaro, L. (2013). Psicología y discapacidad: un encuentro desde el paradigma social. Revista costarricense de psicología, 32, 63 - 74. Recuperado de https://www. google.com.co/?ion $=1 \&$ espv $=2 \# q=$ Dialnet-Psicologia+Discapacidad-4836506\%2520(3).pdf

European Federation of Psychologists' Associations (EFPA). (2016).International Human Rights Day: Psychologists play important role. Recuperado de http://www.efpa.eu/news/december-10international-human-rights-daypsychologists-play-important-role

Fondo de las Naciones Unidas para la Infancia UNICEF (2002). La niñez colombiana en cifras. Oficina de Área para Colombia y Venezuela. Recuperado de https:// www.unicef.org/colombia/pdf/cifras.pdf

González, N. (2008). Convención de las Naciones Unidas sobre los derechos de las personas con discapacidad. Anuario Mexicano de Derecho Internacional, $8,527-540$.

Guba, E. y Lincoln, Y. (1994). Paradigmas que compiten en la investigación cualitativa, Handbook of Qualitative Research, Thousand Oaks, Ca.: Sage. Traducido por Anthony Sampson, Grupo Cultura y Desarrollo Humano. Cali.

Hernández, J.M. (2007). Los Derechos Humanos de las Personas con Discapacidad. Revista quòrum Legislativo, 89, 123. Recuperado de http://www3.diputados. gob.mx/camara/content/view/full/177918
Lázaro, I. (coord.). Halty, A., Meneses, C., Perazzo, C., Roldán, A., y Rúa, A., Uroz, J. (2014). Vulnerabilidad y exclusión en la infancia. Hacia un sistema de información temprana sobre la infancia en exclusión. Cuadernos para el Debate $n^{\circ} 3$, UNICEF Comité Español. Madrid: Editorial Huygens. Recuperado de http://solidaria. unicef.es/pdf/UNICEF_CdebateIII_Vulnerabilidad_y_exclusion_en_la_infanci a_2014.pdf

Ley 1098 de 2006 Corte Constitucional de Colombia (2015). Código de la Infancia y la adolescencia -. Bogotá: Corte Constitucional de Colombia. Recuperado de http://www.corteconstitucional.gov.co/relatoria/CODIGO \%20DE\%20LA \%20INFANCIA $\% 20$ Y\%20LA\%20ADOLESCENCIA.php

Morente, V. (2007). El modelo de la diversidad. La Bioética y los Derechos Humanos como herramientas para alcanzar la plena dignidad en la diversidad funcional [book review] Derechos y Libertades, 17, 231-239. Recuperado de http://orff.uc3m.es/bitstream/handle/10016/8373/ DyL-2007-17-Morente-Modelo.pdf?sequence=1

ONU (2008). Convención Internacional sobre los Derechos de las Personas con Discapacidad. Recuperado de http://www.un.org/esa/socdev/enable/documents/tcconvs.pdf

ONU (2008). Naciones Unidas Enable. El Convenio ya está en vigor ¿Qué sigue? Recuperado de http://www. un.org/spanish/disabilities/Disability_Convention_Fact_Sheet0508spanish.pdf

ONU (2014). Convención sobre los derechos de las personas con discapacidad. Guía de formación Serie de capacitación profesional $N^{o}$ 19. Recuperado de http://www.ohchr.org/ Documents/Publications/CRPD_TrainingGuide_ PTS19_sp.pdf

ONU (2016). Convención sobre los derechos de las personas con discapacidad. Comité sobre los Derechos de las Personas con Discapacidad. Observaciones finales sobre el informe inicial de Colombia. Recuperado de http://www.discapacidadcolombia.com/index.php/colombia-se-raja-en-informe-presentado-a-la-onu

ONU. (2017). Naciones Unidas Enable. Los derechos y la dignidad de las personas con discapacidad. Países y organizaciones de integración regional. Recuperado de http://www.un.org/spanish/disabilities/countries.asp?navid $=18 \&$ pid $=578$ 
Padilla, A. (2010). Discapacidad: contexto, concepto y modelos. International Law: Revista Colombiana de Derecho Internacional, 16, 381-414. Recuperado de http:// www.redalyc.org/articulo.oa?id=82420041012

Palacios, A. (2008). El modelo social de discapacidad: orígenes, caracterización y plasmación en la Convención Internacional sobre los Derechos de las Personas con Discapacidad. Madrid, España: Cinca. Recuperado de http://www.cermi.es/es-ES/ColeccionesCermi/ Cermi.es/Lists/Coleccion/Attachments/64/Elmodelosocialdediscapacidad.pdf

Parra, C.(2010). Convención sobre los Derechos de las Personas con Discapacidad: antecedentes y sus nuevos enfoques. International Law, Revista Colombiana de Derecho Internacional, 16, 347-380.

Romañach, J. y Lobato, M. (2005) Diversidad funcional, nuevo término para la lucha por la dignidad en la diversidad del ser humano. Foro de Vida Independiente.
Recuperado de http://www.forovidaindependiente. org/node/45\#notas

Sanjosé, A. (2007). El primer tratado de derechos humanos del siglo XXI: La Convención sobre los derechos de las personas con discapacidad. Revista electrónica de estudios internacionales, 13, 1-26. Recuperado de http://www. reei.org/reei\%2013/SanjoseGil\%28reei13\%29. pdf

Skliar, C. (2003). ¿Y si el otro no estuviera ahí? Notas para una pedagogía de la diferencia. Madrid: Miño y Dávila.

Schorn, M. (2003). La capacidad en la discapacidad: sordera, discapacidad intelectual, sexualidad y autismo: concepciones psicológicas. Buenos Aires: Lugar.

Villa, N., y Arnau M.S. (2009). Un reto necesario en una sociedad para todas y todos. Las personas con discapacidad en la Universidad. Revista Antena de telecomunicación, 177, 48-54. Recuperado de https://dialnet.unirioja.es/servlet/articulo? codigo $=470812$ 